\title{
Empleo del análisis multivariado en la evaluación de factores no genéticos de cabras Criollas
}

\author{
David, R.N. ${ }^{1}$; Abdala, G.C. ${ }^{1}$; Abdala, M.E. ${ }^{1}$ y Lescano, J.A. ${ }^{1}$
}

'Facultad de Agronomía y Agroindustrias. Universidad Nacional de Santiago del Estero. Santiago del Estero. Argentina.

\section{PalABRAS CLAVE ADICIONALES}

Peso al nacimiento.

Caprinos criollos.

Factores no genéticos.

\section{RESUMEN}

El peso al nacimiento en caprinos es un factor que siempre se pondera en los esquemas productivos por estar positivamente correlacionado con la tasa de crecimiento, con el tamaño del adulto, con el tiempo que tardan las crías en alcanzar peso de faena y con el tipo de alimentación que reciben. El peso al nacimiento varía en las distintas razas por ser determinado genéticamente. Hay productores que llegan a cambiar la raza de cabra buscando mejores pesos al nacimiento y desarrollos más precoces. Existen, sin embargo, factores no genéticos, que influyen en el peso al nacimiento y el desarrollo inicial que pueden llevar a confundir los resultados cuando se comparan diferentes razas buscando la producción de cabritos de mejores pesos al nacimiento y desarrollos más precoces. Sexo y número de camada son dos factores no genéticos relevantes en la determinación del peso al nacimiento y ya han sido demostrados en otras razas. En cabras Criollas, además de estos, se detecta que edad de la madre, número de parto de la madre, y mes de nacimiento también influyen. Se realizó un estudio de la influencia de todas estas variables en el peso al nacimiento y desarrollo inicial en un rebaño caprino de la Universidad Nacional de Santiago del Estero (UNSE), en el que se pesaron crías al nacimiento y en desarrollo inicial en cinco pariciones sucesivas. Se aplicó un análisis conjunto de todas las variables o multivariado, empleándose el método de componentes principales $A C P$, el cual detectó relaciones muy estrechas entre las variables en los tres ejes principales: 1) edad de la madre con número de parto, 2) peso con el tamaño de la camada y 3) otras relaciones, aunque débiles entre sexo y tamaño de camada. El ACP sirvió para sintetizar la información y mejorar la eficiencia en la interpretación de resultados, concentrando a los ejemplares en base a la influencia de las variables estudiadas con sus respectivos pesos al nacimiento, siendo los grupos 2 y 3 los de mayores pesos $(3,18 \mathrm{~kg}$ y $3.4 \mathrm{~kg}$ respectivamente) y los bajos pesos para los grupos 1 y 4 (2,22 kg y $2,25 \mathrm{~kg}$ promedio, respectivamente).

\section{Use of multivariate analysis in the non-genetic factors assessment of Creole goats}

\section{SUMMARY}

In goats, birth weight is a factor that is always taken into account in the production systems since it correlates positively with growth rate, with adult size, with the time required for the offspring reach slaughter weight and with the type of food they receive. Birth weight varies in different races to be genetically determined. There are breeders who change the race of goat focusing on the better birth weight and more early development. However, there are non-genetic factors that influence on the birth weight and early development that can lead to confusing results when comparing different races to looking offspring production of better birth weight and early development. Sex and number of litter are two non genetic factors relevant in determining birth weight, that in addition already it was demonstrated in other races. In Creole goats in addition to these, we found that age of mother, calving number of the mother, and birth month also have influence. A study of the influence of these variables on birth weight and initial development in a goat herd was conducted at the $\mathrm{Na}$ tional University of Santiago del Estero. In this study the offspring were weighed at birth and during early developmental conducted in five successive calving. A pooled analysis of all variables or multivariate analyzes were applied, using the method of principal component $\mathrm{ACP}$, which detect very close relations between the variables in the three major axis: 1) age of mother with calving number, 2) weight with the litter size and 3) other relationships, though weak between sex and litter size. This method, ACP, helped to summarize information and improving efficiency in the interpretation of results, bringing together animals based on the influence of the variables studied and their birth weight. Thus the groups 2 and 3 they presented the higher weights $(3.18 \mathrm{~kg}$ and $3.4 \mathrm{~kg}$ respectively), and groups 1 and 4 , lower weights $(2.22 \mathrm{~kg}$ and $2.25 \mathrm{~kg}$ average respectively).
Cronología del artículo.

Recibido/Received: 05.11.2015

Aceptado/Accepted: 22.02.2016

On-line:11.06.2016

Correspondencia a los autores/Contact e-mail:

rnabdala@unse.edu.ar 


\section{INTRODUCCIÓN}

Santiago del Estero, igual que otras provincias del Noroeste Argentino (NOA), tiene baja densidad de población, con un clima y vegetación del tipo semiárido. Las familias instaladas en el campo disponen por lo general de extensiones bastante amplias de montes con abundante vegetación arbustiva y herbácea perfectamente aptas para la alimentación de las cabras. Las cabras buscan su alimento solas en el monte, regresan a los corrales sin ningún personal que las dirija (Amanquez, 1995).

La producción caprina del NOA, se han orientado tradicionalmente hacia la producción de cabritos, cuya venta o consumo constituyen un importante componente económico para las poblaciones rurales de menores recursos económicos, aprovechando las cabras como productoras de carne, leche o cueros (López de Bava, 1991; Amanquez, 1995).

El sistema de producción usado es el sistema extensivo, donde el ganado aprovecha la flora de los montes naturales disponibles, recibiendo escasa y a veces ninguna suplementación alimentaria y atención sanitaria por parte de los criadores. Este sistema de cría, siempre dependiente de la oferta forrajera de los montes, es fuertemente influenciado por los cambios estacionales que condicionan variaciones en el estado de las flora (Dayenoff, 1991; Legname et al, 2000).

Las cabras poseen la capacidad de crecer en ambientes inhóspitos y con recursos forrajeros escasos (Devendra, 1987), haciendo económicamente aprovechable vastas extensiones de territorios áridos o semiáridos poco aptos para la agricultura o explotación de otro tipo de ganado (Salinas y Dellepiane, 1994), como lo es el Noroeste Argentino. La producción de cabritos lactantes para la venta depende de las cualidades reproductivas de las madres (número de crías e intervalo entre parto, peso al nacimiento, tasa de crecimiento hasta destete, etc.). Existen campañas gubernamentales para cruzar el ganado autóctono con reproductores de otras razas, principalmente Anglo-Nubian, buscando aumentar pesos al nacimiento y de faena, sin evaluar previamente los factores genéticos y no genéticos de las cabras criollas (Legname et al., 1996). Los cruzamientos indiscriminados con la raza Anglo Nubian que se realizaron por toda la provincia han favorecido a productores de las áreas de riego que instalaron tambos caprinos, pero no para mejorar la producción de cabritos en las zonas de secano que son la amplia mayoría de los productores caprinos de la Provincia.

Por otra parte, la introducción indiscriminada de una genética exótica en caprinos locales, ha alterado en forma irreversible la integridad genética de la cabra criolla, que constituía un importante exponente de la biodiversidad ganadera de la región, resultante de siglos de adaptación al medio.

El estudio y conservación de los recursos genéticos es una disciplina emergente, donde la genética es una disciplina clave, en la que intervienen otros componentes de las ciencias puras y aplicadas, por ejemplo la biometría que estudia a los seres vivos (David, 2002).
En los últimos años se desarrolló el análisis multivariado ó multivariante. Según Romero Villafranca (citado por Bramardi, 2000), Desde el punto de vista puramente estadístico y de forma algo restrictiva, los métodos Multivariados pueden definirse como el conjunto de técnicas cuyo objetivo es el análisis descriptivo y/o la realización de inferencias a partir de datos de naturaleza multivariado, es decir, en los que cada observación está constituida por los valores de varias variables interrelacionadas.

Desde el punto de vista práctico las técnicas de análisis multivariado permiten el estudio de situaciones en que se disponen de observaciones sobre varios individuos u objetos pertenecientes a uno o más grupos, estando constituida cada observación por los valores de varias características o variables. Los conceptos de individuo, grupo y variable pueden corresponder a realidades muy diferentes según el problema considerado (Bramardi, 2000).

Desde el punto de vista de las aplicaciones, los criterios más difundidos para clasificar las distintas técnicas del análisis multivariado son la naturaleza de las matrices de datos analizadas y los objetivos perseguidos en el análisis.

Se trata de los métodos llamados multidimensionales, por oposición a los métodos de estadística descriptiva, que no tratan más que de una o dos variables a la vez. Por lo tanto, permiten la confrontación entre numerosas informaciones, lo que es mucho más provechoso que su examen por separado. Extraen las tendencias más sobresalientes de datos demasiado numerosos, jerarquizándolos y eliminando los efectos marginales o puntuales que perturban la percepción global de los hechos (Escofier y Pagès, 1992).

Los métodos multivariados que conciernen al presente trabajo resuelven el problema de representar geométricamente y de cuantificar la asociación entre individuos, como así también de clasificarlos respecto a un conjunto de variables y/o índices cuantitativos observados.

Uno de los métodos multivariados, es el análisis factorial que involucra al análisis de componentes principales (ACP). El análisis factorial se utiliza sólo o conjuntamente con métodos de clasificación, mientras que estos últimos apenas se utilizan solos. Ocupa un lugar primordial entre los métodos de análisis de datos, fundamentalmente debido a las representaciones geométricas de los mismos que transforman en distancias euclideas las proximidades estadísticas entre los elementos (Escofier y Pagès, 1992).

El ACP describe aspectos observables y el resultado proporcionado por dicho análisis consiste en presentar los datos, sin hacer ninguna suposición sobre estructuras subyacentes inobservables. Se busca reducir la información, pasando de un conjunto de variables a otro más reducido que representa a las primeras, sin hacer ninguna hipótesis sobre el significado de los factores. Lo que interesa es describir cuales son las dimensiones principales (Hair et al., 1999; Bramardi, 2000; David, 2002).

Los componentes principales se pueden obtener mediante la matriz de variancias-covariancias entre 
variables y la matriz de correlaciones. Los resultados a los que se arriba por uno u otro método son distintos y la elección del tipo de matriz de entrada es un tema controvertido. Si las unidades de medida de las variables son diferentes, es preferible el uso de la matriz de correlaciones porque equivale a utilizar variables reducidas y por lo tanto, sin dimensión física. Pero si las unidades de medida son las mismas o razonablemente conmensurables, es preferible realizar el estudio sobre la matriz de varianzas-covariancias, que es menos artificial (Bramardi, 2000).

El propósito del presente trabajo fue valorar la importancia de los factores no genéticos en el peso al nacimiento de caprinos criollos verificando mediante la técnica del ACP, la existencia de acciones conjuntas de los mismos y determinando las condiciones básicas de las madres para obtener mejores pesos al nacimiento sin realizar cambios genéticos, con la ayuda del paquete estadístico SPADN Versión 3.5 (Crivisqui, 1998).

\section{MATERIAL Y MÉTODOS}

El presente trabajo se realizó sobre un rodeo caprino, en la cabaña experimental de la Facultad de Agronomía de la UNSE, ubicada en la localidad de El Zanjón (Dpto. Capital), distante a 15 km aproximadamente de la de la ciudad capital de Santiago del Estero.

La cabaña cuenta con una superficie de 250 hectáreas aproximadamente, las cuales en su mayoría se encuentra con monte nativo siendo la principal fuente de alimentación de los animales.

Dicha explotación caprina está localizada en el Área de Riego de la Provincia, aproximadamente entre los $27^{\circ}$ y $29^{\circ}$ de Latitud Sur, $64^{\circ}$ y $62^{\circ}$ de Longitud Oeste y a una Altitud que varía entre 100 y 200 metros sobre el nivel del mar.

Los suelos son originados de los sedimentos aluvio-eólicos, caracterizados por poseer textura media, prevaleciendo las arenas finas y limo, y de escaso desarrollo, definiendo de esta manera al perfil del tipo A-AC-C (entisoles); estas características le confieren al mismo un excesivo drenaje y poca retención de humedad (Duffou, 1981).

El 60\% del total del área de riego del río Dulce está afectada por problemas de salinización y sodificación, por lo que restringe a la actividad agrícola. Estos factores limitantes se deben principalmente a la presencia de napas freáticas salinas, drenaje natural restringido, uso irracional de agua de riego, presencia de canales sin revestimiento que aportan a la freática.

La provincia pertenece a la región fitogeográfica del Chaco semiárido, en la que se distinguen dos estaciones opuestas: la estación lluviosa y de fuertes calores (primavero-estival) que abarca desde octubre hasta marzo y la estación seca de moderada temperatura (otoño-invernal) que se prolonga desde abril hasta septiembre. El promedio de lluvias anuales se encuentra alrededor de $575 \mathrm{~mm}$, percibiéndose un decrecimiento desde el este al oeste. Los valores de ocurrencia de granizo, son de baja de 4 a 5 granizadas con intensidad variable en términos de 10 años, siendo los meses de octubre a enero los de mayor probabilidad (Torres Bruchmann, 1981).
Relacionando las precipitaciones y la evaporación de la provincia se puede establecer el balance hídrico de la misma, observándose que existe una marcada deficiencia hídrica, que aparece en los doce meses del año, siendo más pronunciada en verano y más suave durante el invierno (Torres Bruchmann, 1981).

Las temperaturas son del tipo continental, cálidas en verano y frío en invierno. La temperatura media anual es de $27^{\circ} \mathrm{C}$. En verano se presentan temperaturas madias entre 26 y $28^{\circ} \mathrm{C}$, con una máxima absoluta de hasta $47^{\circ} \mathrm{C}$. En invierno se registran temperaturas medias que van desde 12 a $14^{\circ} \mathrm{C}$ con una mínima absoluta de hasta $-10^{\circ} \mathrm{C}$ (Torres Bruchmann, 1981).

Las heladas se presentan en la segunda mitad de otoño y las últimas a mediado del invierno, pero existe la probabilidad de ocurrencia de heladas extremas una cada 50 años, las heladas tempranas se pueden dar a partir del 24 de abril y las tardías hasta el 28 de septiembre (Torres Bruchmann, 1981).

Dada la importancia de la producción de cabritos para la provincia y buscando contribuir a una productividad más eficiente en las cabras criollas, se analizaron durante cinco años los factores biológicos que inciden más directamente en la producción de cabritos en un rebaño criadas en un campo experimental de la UNSE. El rebaño fue criado en un sistema extensivo, con las cabras alimentadas con el libre ramoneo de la flora nativa, y con padrillos acompañando en forma permanente la majada con servicio libre, simulando las condiciones de cría de la mayoría de los productores de las zonas de secano.

Se pesaron caprinos al nacimiento y cada diez días durante los dos primeros meses de desarrollo de un rebaño experimental de caprinos criollos de la UNSE, compuesto por 40 hembras y 3 machos manejados en un sistema de cría semi-extensiva con servicio libre. Se tomaron datos de los peso al nacimiento y en desarrollo de 156 pariciones. También se registraron edad, número de parto, mes y año de parición de las madres. Los pesos se determinaron mediante una balanza tipo pilón de $20 \mathrm{~kg}$ con una precisión de $0,05 \mathrm{~kg}$ en horas de la mañana. El peso al nacimiento fue determinado siempre a menos de 24 horas del parto.

Para estudiar la variabilidad entre los ejemplares caprinos y su grado de similitud y la asociación entre las variables se utilizó el análisis de componentes principales (ACP). Este análisis busca reducir la dimensión del espacio en el cual están representadas las variables originales, que en este caso son cuantitativas, para detectar estructuras de variabilidad y, eventualmente, realizar agrupaciones por similitud (Bramardi, 2000).

\section{RESULTADOS}

El ACP fue realizado a partir de una matriz de datos involucrando 156 individuos (o filas) y 6 variables o columnas (sexo, tamaño de la camada, edad de la madre, número del parto, mes y peso al nacimiento).

Dado que una de las propiedades del ACP es mostrar el sentido de relación entre las variables y el grado de asociación entre ellas, las 6 variables fueron consideradas como variables activas. Estas no poseían las mismas unidades de medición y por lo tanto el ACP fue realizado a partir de los datos centrados y reducidos (Carrasco de la Peña y Huerta, 1993). 
Tabla I. Valores propios y contribución a la variancia total (absoluta y acumulada) entre las variables (Eigenvalues and contribution to the total variance (absolute and accumulated) between the variables).

\begin{tabular}{lccc}
\hline $\begin{array}{l}\text { Variables } \\
\text { Ejes }\end{array}$ & Valores propios & $\begin{array}{c}\text { Contribución sobre la variancia total } \\
\text { (valor absoluto) }\end{array}$ & $\begin{array}{c}\text { Contribución sobre la variancia total } \\
\text { (valor acumulativo) }\end{array}$ \\
\hline Eje 1 & 2,2815 & 38,02 & 38,02 \\
Eje 2 & 1,3111 & 21,85 & 59,88 \\
Eje 3 & 0,9727 & 16,54 & 76,42 \\
\hline
\end{tabular}

La segunda columna muestra los primeros valores propios, o sea la varianza sobre los ejes principales que superan la unidad. La tercera columna indica los porcentajes de contribución para cada uno de los ejes principales. La cuarta columna muestra los porcentajes de contribución acumulativos para los distintos ejes.

Se uso el criterio de selección para los componentes principales establecido por Káiser (citado por Bramardi, 2000), el cual se basa en la elección de los componentes principales cuyos valores (pesos) propios superan la unidad, las cuales según este autor son los mejor representados. Dado que el ACP pretende evaluar la posible similitud entre los ejemplares caprinos, todos los animales fueron considerados como individuos activos.

Para conocer la calidad de dicha representación gráfica, fue necesario tener en cuenta el coseno al cuadrado $\left(\cos ^{2}\right)$ de los ángulos formados por los valores de los ejemplares con cada uno de los componentes principales. El $\cos ^{2}$ es un buen indicador de la calidad de la proyección del individuo en el plano. Según indica Philippeau (1986) un individuo está bien representado en el plano, cuando la suma de dichos $\cos ^{2}$ es mayor a 0,5.

Al factorizar la matriz simétrica de $6 \times 6$ variables que guarda las correlaciones entre las columnas de la matriz rectangular de información original se obtiene que los dos primeros ejes principales (que superan la unidad) explican el 59,88\% del total de la variación entre los caprinos. En la siguiente tabla, se presenta a modo de resumen la contribución a la variancia total en valores absoluto y acumulativo para cada uno de los ejes o Componentes Principales (CP).

De acuerdo con los datos de la tabla I, los valores absolutos de contribución del primer eje sobre la varianza total es de $38,02 \%$, mientras que para el tercero es $16,54 \%$. En este último eje el valor acumulado de información generada por los caprinos fue del 76,42\% de la varianza total. Esto implica que más de las tres cuartas partes de la variabilidad total entre los 156 caprinos evaluados están representados en los primeros 3 ejes principales.

En la tabla II, se muestran las saturaciones o correlaciones que existen entre las 6 variables originales y los tres primeros componentes principales a fin de interpretar los gradientes subyacentes en ellos.

Las variables que intervienen en la construcción del primer componente resaltan por su magnitud las variables maternas principalmente: edad de la madre y número de parto y en menor medida, mes de nacimiento. Estas variables son las que más han contribuido a la variación de éste eje, por lo que el componente principal Uno, podría entonces ser considerado como condición materna y época de nacimiento. Las dos primeras variables tienen un coeficiente de correlación negativo con el eje 1, lo que estaría determinando en el plano un gradiente de izquierda a derecha.

La contribución más importante al segundo eje, pero de mediana representación, la hacen las variables correspondientes a la tamaño de camada y peso al nacimiento. Por lo que el segundo componente principal podría ser interpretado como tamaño de la camada y peso de la cría. Aquí también debe destacarse el aporte negativo de la variable tamaño de la camada estableciéndose un gradiente de aumento de tamaño, de abajo hacia arriba en los casos que el eje 2 sea utilizado como ordenada en el plano, mientras que el peso al nacimiento proporciona un aporte positivo por lo que en este caso el gradiente se establece desde arriba hacia abajo.

En cuanto al tercer eje, el coeficiente de correlación de la variable sexo es la que presenta los valores más altos, por lo tanto es la única característica mejor asociada a éste eje. En consecuencia el tercer componente principal puede ser interpretado como sexual, con aporte negativo por lo que en este caso el gradiente se efectúa desde arriba hacia abajo.

Sobre un total de 156 caprinos analizados, el número y el porcentaje de ejemplares que se encuentran bien proyectadas en los distintos planos $\left(\cos ^{2}>0,50\right)$ son los siguientes:

$$
\begin{aligned}
& \text { Plano } 1-2=84 \text { caprinos }-53,85 \% \\
& \text { Plano } 1-3=74 \text { caprinos }-47,44 \% \\
& \text { Plano } 1-4=50 \text { caprinos }-32,05 \%
\end{aligned}
$$

En la siguiente figura se proyectan simultáneamente los ejemplares caprinos en el plano 1-2, y las variables observadas sobre ellos, basado en un gráfico bidimensional o biplot (Kempton, 1984), a los efectos de mostrar el comportamiento de los animales en función de las coordenadas del ACP.

El estudio de las variables (Escofier y Pages, 1992) indica que los caprinos que están bien representados se ubican a la izquierda de la CP1 $\left(2^{\circ}\right.$ y $3^{\circ}$ cuadrante), provienen de madres de mayor edad con mayor número de partos, que paren en los meses de abril y mayo. Los ubicados a la derecha $\left(1^{\circ}\right.$ y $4^{\circ}$ cuadrante) tienen la expresión opuesta. Los que se ubican debajo de la CP2 ( $3^{\circ}$ y $4^{\circ}$ cuadrante) tienen partos numerosos y bajo peso al nacimiento, por el contrario, los que se posicionen hacia arriba serán de mayor peso pero provendrán de partos simples. 
Tabla II: Coeficientes de correlación (r) entre las variables cuantitativas y los cuatro primeros ejes principales (Correlation coefficients ( $r$ ) between quantitative variables and the first four main axes).

\begin{tabular}{|c|c|c|c|c|}
\hline \multirow[b]{3}{*}{ Variables } & \multirow{3}{*}{$\begin{array}{l}\text { Componentes } \\
\text { Principales }\end{array}$} & \multicolumn{3}{|c|}{ Correlaciones variables - ejes } \\
\hline & & Eje 1 & Eje 2 & Eje 3 \\
\hline & & $r$ & $\mathrm{r}$ & $\mathrm{r}$ \\
\hline Sexo & & 0,39 & $-0,23$ & $-0,57$ \\
\hline Tamaño de la camada & & $-0,13$ & $-0,78$ & 0,49 \\
\hline Edad de la madre & & $-0,92$ & $-0,20$ & $-0,30$ \\
\hline Número de parto & & $-0,89$ & $-0,24$ & $-0,32$ \\
\hline Mes de nacimiento & & 0,49 & $-0,12$ & $-0,48$ \\
\hline Peso al nacimiento & & $-0,47$ & 0,74 & 0,08 \\
\hline
\end{tabular}

Las variables están muy bien representadas cuando $r>0,80$; bien representadas: $0,65 \leq r \leq 0,80$; y medianamente bien representadas cuando $r<0,65$ (Philippeau, 1986).

La figura 1 muestra además los 5 grupos que se forman en el plano factorial 1-2, los cuales presentan las siguientes características:

Grupo 1: integrado por 39 caprinos (25\% del total) que se posicionaron en el $1^{\circ}$ y $4^{\circ}$ cuadrante. De ellos 25 están bien representados, es decir el $\cos ^{2}>$ a 0,5 . Estos ejemplares se caracterizan por tener las madres más jóvenes del rodeo, con 1 a 2 partos y con crías con los más bajos pesos al nacimiento. Las crías son de sexo femenino y sus partos ocurrieron en el $2^{\circ}$ semestre del año (julio y agosto).

Grupo 2: los individuos se posicionan en la parte superior del plano influenciados por las características que contribuyen el eje 2 (por arriba del eje 1 y a la derecha del eje 2), en el $1^{\circ}$ cuadrante. Está integrado por 23 ejemplares (aproximadamente 15\%). De ellos se desta- can 7 animales por su buena representación en el plano 1-2. Son animales que poseen alto peso al nacimiento, superiores a $3 \mathrm{~kg}$ pero provienen de madres que dieron una sola cría por parto. Son madres jóvenes de 1 a 3 años de edad.

Grupo 3: integrado por 37 animales (aproximadamente $24 \%$ ) que se ubican en la parte superior del plano, en el $2^{\circ}$ cuadrante. Se caracterizan por variables relacionadas al peso al nacimiento, el mes de nacimiento y el tipo de sexo. De los 37 ejemplares 20 están bien representados en el plano, ya que superan el valor de 0,5 del $\cos ^{2}$. Son los de mayor peso al nacimiento. La edad de las madres oscila entre los 3 y 5 años. Al igual que el Grupo 2, son ejemplares que provienen de partos simples pero además son ejemplares de sexo masculino prácticamente en su totalidad.

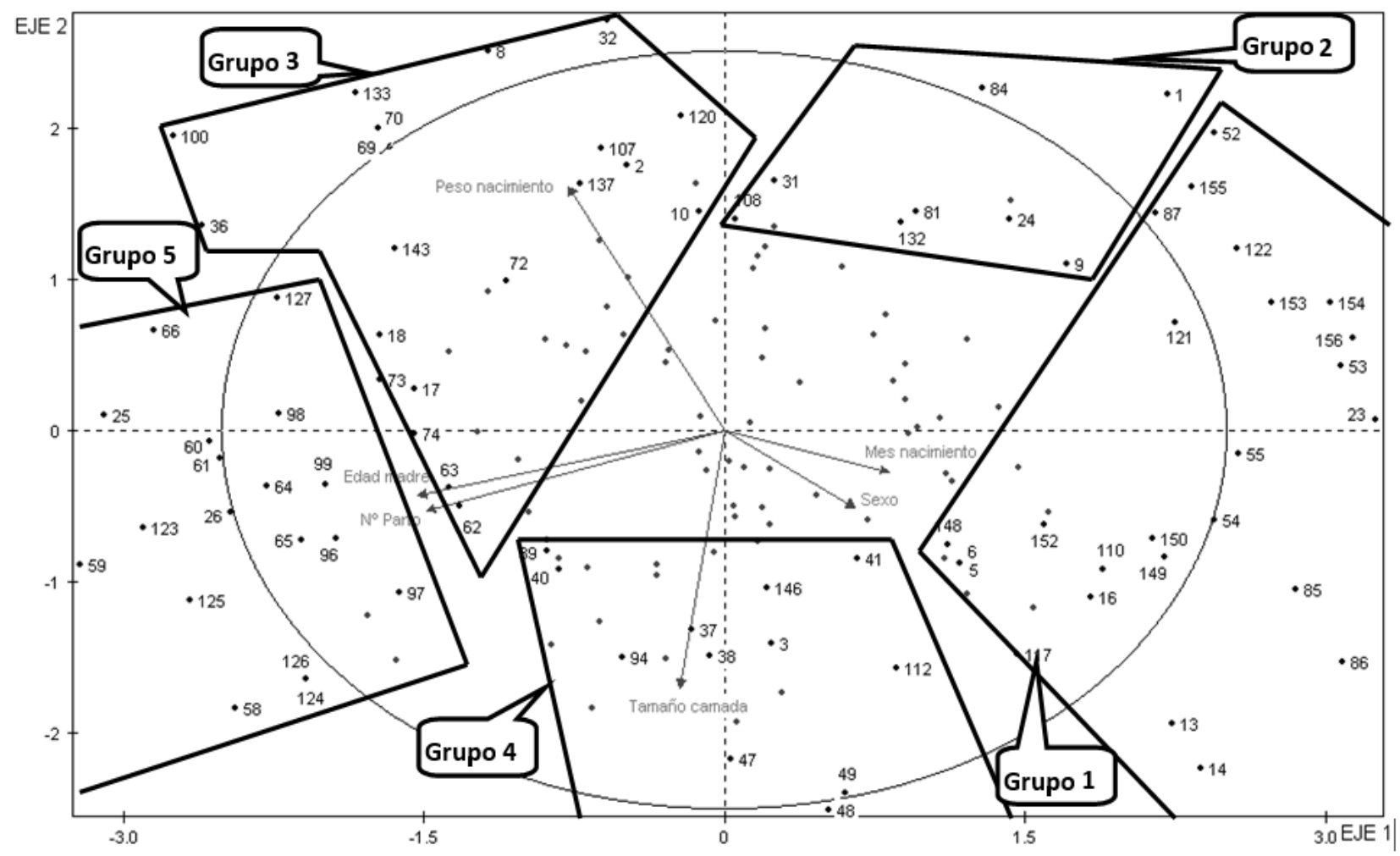

Figura 1. Representación gráfica en biplot de los caprinos bien representados y las variables sobre ellos observadas en el Plano 1-2 (Graphical representation on biplot of caprines well represented and observed variables over them in the Plane 1.2). 
Grupo 4: son los caprinos que se posicionan en la parte inferior del plano en los cuadrantes $3^{\circ}$ y $4^{\circ}$, influenciados por las características de la componente principal 2. Se caracterizan por provenir de partos múltiples con bajo peso al nacimiento. Las madres tienen 3 o más años y parieron entre los meses de junio y julio.

Grupo 5: este grupo se caracteriza por que sus ejemplares provienen de madres de mayor edad del rodeo, con cinco o más años de edad. Las mismas tienen partos múltiples. Estos caprinos tienen peso al nacimiento igual o menor al promedio general.

\section{DISCUSIÓN}

El análisis de componentes principales (ACP) agrupa a los ejemplares con base a la influencia de los factores en consideración en su respectivo peso al nacimiento. Presenta además la ventaja de incluir en los agrupamientos solamente a los ejemplares que se encuentran bien representados.

La ubicación de los individuos en las distintas regiones del plano teniendo como referencias a los componentes principales, determina agrupamientos donde se encuentran los individuos con los mayores pesos al nacimiento (promedios 3,18 $\mathrm{kg}$ y 3,4 $\mathrm{kg}$ para los grupos 2 y 3 respectivamente), y agrupamientos donde se reconocen pesos al nacimiento bajos $(2,22 \mathrm{~kg}$ y $2,25 \mathrm{~kg}$. promedio para los grupos 1 y 4).

La variación del peso al nacimiento es grande, quedando señaladas las direcciones en las cuales la variación se dirige hacia el aumento de peso al nacimiento o hacia su disminución.

Los factores analizados con el ACP justifican el $76,42 \%$, lo que significa que el total de la variabilidad ambiental es aún mayor, dado que no se tienen en cuenta otros factores como estado fenológico de las cabras, el año de parición, entre otros.

Dada variabilidad tan amplia de origen no genético en el peso al nacimiento de caprinos, y como se evalúa exclusivamente con cabras criollas, cuando se realizan cruzamientos con otras razas, buscando incrementar los pesos al nacimiento, como se realiza en la actualidad en varias provincias de la Argentino; serían necesarios factores de corrección más rigurosos para descartar la influencia de los factores no genéticos o ambientales en el peso al nacimiento de las cabras criollas que se usan como raza básica para el cruzamiento, para así poder valorar las ganancias de peso reales obtenidas como resultado del cruzamiento interracial.

\section{AGRADECIMIENTOS}

Los autores agradecen al Consejo de Investigación de Ciencia y Técnica, dependiente de la Universidad Nacional Santiago del Estero - Argentina, por el financiamiento de los sendos Proyectos de Investigación en la temática caprina, que permitieron por más de quince años, recabar la información necesaria para la concreción de este trabajo entre otros.

También agradecemos profundamente al Dr. César René Legname por la ayuda brindada en forma desinteresada, en la confección de éste trabajo, en la asesoría científica y en la recolección de datos.

\section{BIBLIOGRAFÍA}

Amanquez, J.C. 1995. La problemática actual del ganado caprino en Santiago del Estero. Tesis de Lic. en Biología. FAyA - UNSE. Santiago del Estero. Argentina.

Bramardi, S.J. 2000. Estrategias para el análisis de datos en la caracterización de recursos naturales. Tesis doctoral. Universidad Politécnica de Valencia. Departamento de Estadística e Investigación Operativa. Valencia. España.

Carrasco de la Peña, J.L. y Huerta, M.A. 1993. Estadística multivariante en las ciencias de la vida. Edit. Ciencia 3. S.L. Madrid. España.

Crivisqui, E.M. 1998. Laboratoire et méthodologie du traitement de données. Programme PRESTA. Programme de Richerche et Dénseignemen en Estatistique Appliquée. SPADN.

David, R.N. 2002. Caracterización morfológica y agronómica de plantas híbridas de cítricos de la EEA. Tesis de Magister Scientiae. Unidad Integrada FCA. INTA. UNMdP. Famaillá. Tucumán. Argentina.

Dayenoff, P.Ñ.; Caceres, R.; Carrizo, H. y Bolaño, M. 1991. Peso al nacimiento y crecimiento del cabrito tipo Criollo regional. Ed. INTA. E.E.A. La Rioja. Argentina.

Devendra, C. 1987. The role of goats in food production systems in industrialised and developing countries. Proceedings of the IV International Conference on Goats. Brasilia.

Duffou, R. 1981. Caracterización de los suelos de la provincia de Santiago del Estero. Dirección de Minería y Geología. Ministerio de Economía. Gobierno de la Provincia de Santiago del Estero. Argentina

Escofier, B. y Pages, J. 1992. Análisis factoriales simples y múltiples. Objetivos, métodos de interpretación. Ed. Universidad del País Vasco. Bilbao.

Hair, J.F.; Anderson, R.E.; Tatham, R.L. y Black, W. 1999. Análisis multivariante. Ed. Prentice Hall. Madrid.

Kempton, R.A. 1984. The use of biplot in interpreting variety by environmental interactions. J Agric Sci. 103: 123-135.

Legname, C.R.; David, R.N. y Moggio, P. 1996. Peso y dimensiones corporales de caprinos regionales de Santiago del Estero. Rev Arg Prod Anim, 16 (suplemento 1).

Legname, C.R. y David, R.N. 2000. Peso y dimensiones corporales de cabras Criollas de Santiago del Estero. Rev Cienc Tecnol, Serie Científica UNSE.

López de Bava, S. 1991. La cría de caprinos en Santiago del Estero (Argentina). Situación actual y perspectivas. Tesis de Magíster en Ciencias Agrarias. Facultad de Agronomía. Univ. Georgia Augusta Gottingen. Alemania.

Philippeau, G. 1986. Comment interprèter les résultats d'une analyse en composantes principales. Edit. TCFF. Paris.

Salinas, A. y Dellepiane, G. 1994. Situación del Sector Caprino Nacional. Secretaria de Agricultura, Ganadería y Pesca (SAGyP). Argentina.

Torres Bruchmann, E. 1981. Climatología general y agrícola de la provincia de Santiago del Estero. Ed. Departamento de Ecología. Fac. de Agronomía y Zootecnia. UNT. 